\title{
Table of Cases
}

\author{
U.S. CASES
}

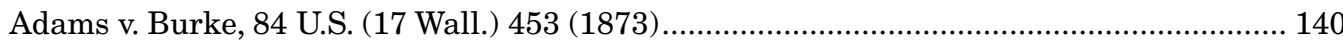

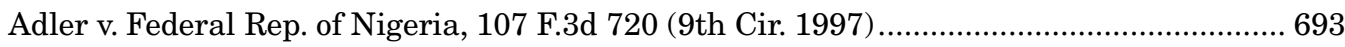

Al Haramain Islamic Found., Inc. v. U.S. Department of Treasury, 585 F.Supp.2d 1233 (D. Or. 2008).

Al Haramain Islamic Found., Inc. v. U.S. Department of Treasury, 660 F.3d 1019 (9th Cir. 2011) 406

AMCO UKRSERVICE v. AMERICAN METER CO., 312 F.Supp.2d 681 (E.D. Pa. 2004) 205

Atlantic Marine Const. Co. v. U.S. Dist. Ct. for W. Dist. of Tex., 571 U.S. 49 (2013). 664

Baloco v. Drummond Co., 631 F.3d 1350 (11th Cir. 2011).... 526

BANCO GENERAL RUNIÑAHUI, S.A. v. CITIBANK INT'L, 97 F.3d 480 (11th Cir. 1996) 276

Banco Nacional de Cuba v. Sabbatino, 376 U.S. 398 (1964) 697

Bauman v. DaimlerChryster Corp., 644 F.3d 909 (9th Cir. 2011). 507

Beltronics USA Inc. v. Midwest Inventory Distrib. LLC, 90 U.S.P.Q.2d 1228 (10th Cir. 2009) 165

BERGESEN v. JOSEPH MULLER CORP., 710 F.2d 928 (2d Cir. 1983) 721

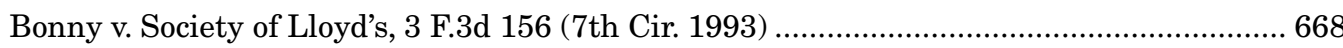

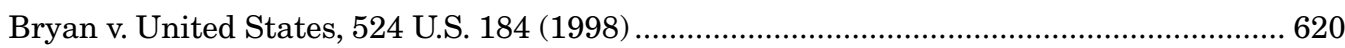

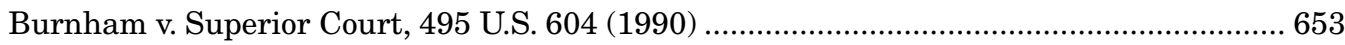

Calhoun v. Yamaha Motor Corp., U.S.A., 40 F.3d 622 (3d Cir. 1994) .................................... 260

Chevron U.S.A. Inc. v. Natural Resources Defense Council, Inc., 467 U.S. 837 (1984) ......... 452

Commercial Union Ins. Co. v. Sea Harvest Seafood Co., 251 F.3d 1294 (10th Cir. 2001) ..... 260

Crosby v. National Foreign Trade Council, 530 U.S. 363 (2000) .............................................. 10

Doe VIII v. Exxon Mobil Corp., 654 F.3d 11 (D.C. Cir. 2011) .................................................. 549

ELI LILLY DO BRASIL, LTDA v. FEDERAL EXPRESS CORP., 502 F.3d 78 (2d Cir. 2007) 670

Equal Emp’t Opportunity Comm'n v. Arabian Am. Oil Co., 499 U.S. 244 (1991)............178, 756 Ferrostaal Metals Corp. v. United States, 664 F. Supp. 535 (Ct. Int'l Trade 1987) ............... 329 Flomo v. Firestone Natural Rubber Co., 643 F.3d 1013 (7th Cir. 2011)............................... 507

Fuller Co. v. Compagnie des Bauxites de Guinée, 421 F. Supp. 938 (W.D. Pa. 1976) ............ 717 Goodyear Dunlop Tires Operations, S.A. v. Brown, 564 U.S. 915 (2011) ................................ 652 Grupo Gigante SA de CV v. Dallo \& Co., Inc., 391 F.3d 1088 (9th Cir. 2004)........................ 792 
Hale v. Co-Mar Offshore Corp., 558 F. Supp. 1212 (W.D. La. 1984) ...................................... 678

HARTFORD FIRE INS. CO. v. CALIFORNIA, 509 U.S. 764 (1993) ................................. 768

HIH Marine Services, Inc. v. Fraser, 211 F.3d 1359 (11th Cir. 2000)...................................... 262

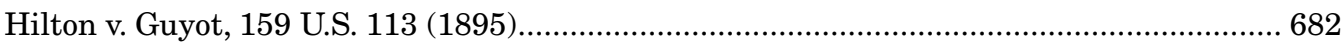

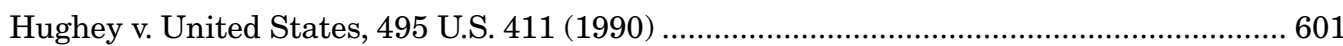

Impression Prods. Inc. v. Lexmark Int'l Inc., 581 U.S. _, 137 S. Ct. 1523 (2017) ................. 178

Ingersoll Milling Machinery Co. v. M/V Bodena, 829 F.2d 293 (2d Cir. 1987)...................... 260

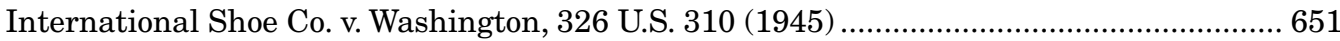

International Thunderbird Gaming Corp. v. United Mex. States, 473 F.Supp.2d 80

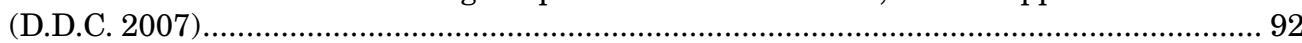

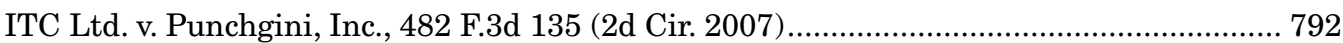

Jazz Photo Corp. v. International Trade Commission, 264 F.3d 1094 (Fed. Cir. 2001) ......... 178

JESNER v. ARAB BANK, PLC, 584 U.S. _, 138 S.Ct. 1386 (2017) ................................550

Jones v. Sea Tow Serv. Freeport NY Inc., 30 F.3d 360 (2d Cir. 1994) .................................. 720

Karl Koch Erecting Co. v. New York Convention Center Dev. Corp., 838 F.2d 656 (2d

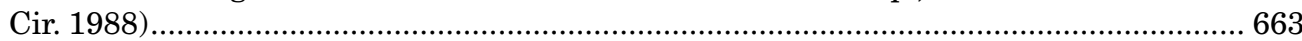

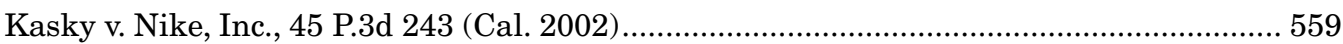

Kiobel v. Royal Dutch Petroleum Co., 569 U.S. 108 (2013)................................................. 549

KIRTSAENG v. JOHN WILEY \& SONS, INC., 568 U.S. 519 (2013) ............................. 167

Klaxon v. Stentor Electric Mfg. Co., 313 U.S. 487 (1941)....................................................... 679

Lander Co. v. MMP Investments, Inc., 107 F.3d 476 (7th Cir. 1996) ................................... 649

LEVER BROTHERS CO. v. UNITED STATES, 877 F.2d 101 (D.C. Cir. 1989) ............. 156

Lever Brothers Co. v. United States, 981 F.2d 1330 (D.C. Cir. 1993) ...................................... 163

Litecubes LLC v. Northern Light Products, Inc., 523 F.3d 1353 (Fed. Cir. 2008) .................. 797

LONDON FILM PRODS. v. INTERCONTINENTAL COMMC'NS, 580 F. Supp.

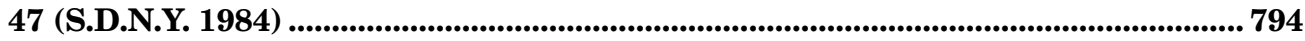

M/S BREMEN v. ZAPATA OFF-SHORE CO., 407 U.S. 1 (1972) ..................................... 657

Maison Prunier v. Prunier's Restaurant \& Café, 288 N.Y.S. 529 (N.Y. Sup. Ct. 1936) ......... 792

Mars Inc. v. Kabushiki-Kaisha Nippon Conlux, 24 F.3d 1368 (Fed. Cir. 1994) ..................... 776

McBEE v. DELICA CO., 417 F.3d 107 (1st Cir. 2005) .................................................. 783

MCC-MARBLE CERAMIC CTR. v. CERAMICA NUOVA D'AGOSTINO, 144

F.3d 1384 (11th Cir. 1998) ............................................................................................................ 217

Medellin v. Texas, 552 U.S. 491 (2008) ................................................................................. 4

Mercury Coal \& Coke, Inc. v. Mannesmann Pipe \& Steel Corp., 696 F.2d 315 (4th Cir. 1982). 663

Mid-America Tire, Inc. v. PTZ Trading Ltd., 768 N.E.2d 619 (Ohio 2002) ........................... 275

Mitsubishi Motors Corp. v. Soler Chrysler-Plymouth, Inc., 473 U.S. 614 (1985) ................... 728

MORRISON v. NATIONAL AUSTRALIA BANK, 561 U.S. 247 (2010) .................... 178, 806

Nedlloyd Lines B.V. v. Superior Court, 834 P.2d 1148 (Cal. 1992)........................................ 678

Office of Foreign Assets Control v. Voices in the Wilderness, 329 F.Supp.2d 71 (D.D.C. 2004)...... 


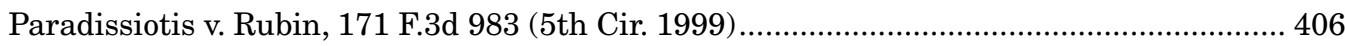

Pelleport Investors Inc. v. Budco Quality Theatres Inc., 741 F.2d 273 (9th Cir. 1984) ......... 663

Pinker v. Roche Holdings Ltd., 292 F.3d 361 (3d Cir. 2002) .................................................... 753

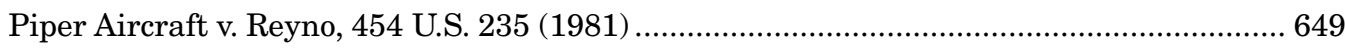

PPG Indus. v. Pilkington plc, 825 F. Supp. 1465 (D. Ariz. 1993) …........................................ 712

Prutscher v. Fidelity Int'l Bank, 502 F. Supp. 535 (S.D.N.Y. 1980) ..................................... 275

Quality King Distributors v. L'anza Research Int'l, 523 U.S. 135 (1998) ............................. 166

Ralls Corp. v. Committee on Foreign Inv. in the U.S., 758 F.3d 296 (D.C. Cir. 2014) ............ 526

Rent-A-Center, West, Inc. v. Jackson, 561 U.S. 63 (2010) ....................................................... 711

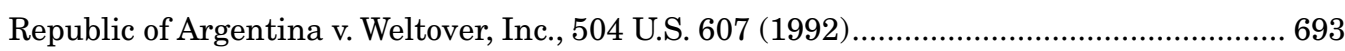

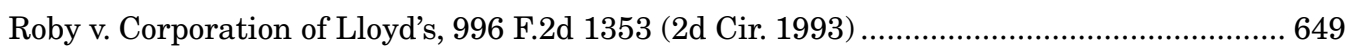

Sakar Int'l, Inc. v. United States, 516 F.3d 1340 (Fed. Cir. 2008) ........................................ 140

Samantar v. Yousuf, 560 U.S. 305 (2010)......................................................................172, 692

SCHAPER MFG. CO. v. U.S. INT'L TRADE COMM'N, 717 F.2d 1368 (Fed. Cir.

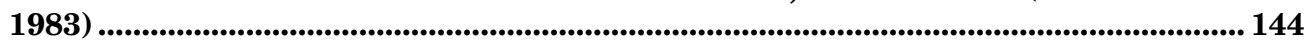

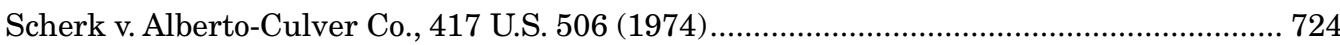

Shahan v. Shahan, 988 S.W.2d 529 (Mo. 1999) (en banc)................................................. 260

SKF USA Inc. v. International Trade Comm'n, 423 F.3d 1307 (Fed. Cir. 2005) ..................... 164

Société Nationale Industrielle Aérospatiale v. U.S. Dist. Ct., 482 U.S. 522 (1987) ............... 682

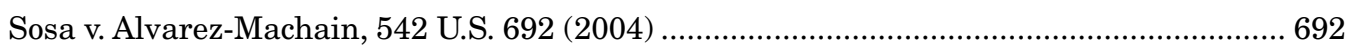

Spanski Enterprises, Inc. v. Telewizja Polska, S.A., 883 F.3d 904 (D.C. Cir. 2018) ................ 797

Spectrum Stores, Inc. v. Citgo Petroleum Corp., 632 F.3d 938 (5th Cir. 2011) ..................... 698

STEELE v. BULOVA WATCH CO., 344 U.S. 280 (1952).............................................. 777

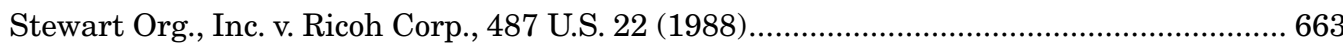

SUPERIOR WIRE v. UNITED STATES, 867 F.2d 1409 (Fed. Cir. 1989) ....................... 327

Tire Eng'g \& Distribution, LLC v. Shandong Linglong Rubber Co., 682 F.3d 292 (4th

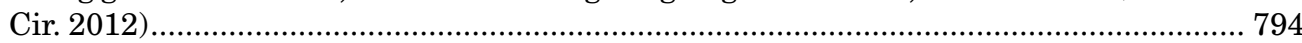

TORRINGTON CO. v. UNITED STATES, 474 F. Supp. 744 (Ct. Int'l Trade 1990) .... 446

Torrington Co. v. United States, 938 F.2d 1278 (Fed. Cir. 1991) .......................................... 452

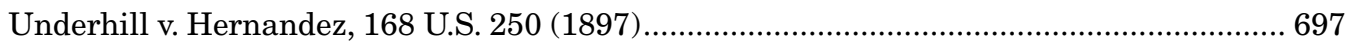

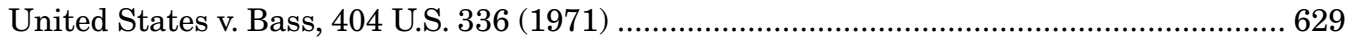

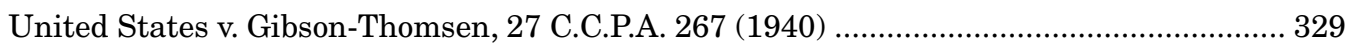

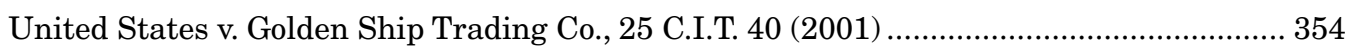

United States v. Gorman, 807 F.2d 1299 (6th Cir. 1986) ......................................................... 619

United States v. Hsieh Hui Mei Chen, 754 F.2d 817 (9th Cir. 1985) .................................... 619

UNITED STATES v. KAY, 359 F.3d 738 (5th Cir. 2004) ................................................... 622

UNITED STATES v. KOZENY, 582 F.Supp.2d 535 (S.D.N.Y. 2008) ................................ 631

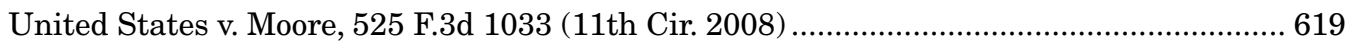

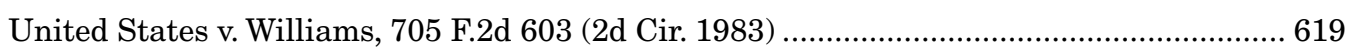

Vaudable v. Montmartre, 193 N.Y.S.2d 332 (N.Y. Sup. Ct. 1959) .......................................... 792

xiv 
Ventress v. Japan Airlines, 486 F.3d 1111 (9th Cir. 2007).................................................. 507

Vimar Seguros y Reaseguros, S.A. v. M/V Sky Reefer, 515 U.S. 528 (1995)........................... 729

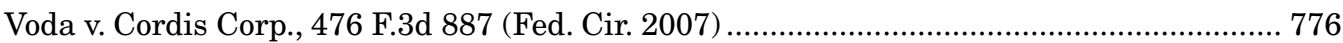

W.S. Kirkpatrick \& Co. v. Environmental Tectonics Corp., 493 U.S. 400 (1990) ................... 697

Wilburn Boat Co. v. Fireman's Fund Ins. Co., 348 U.S. 310 (1955) ........................................ 259

WILSON v. LIGNOTOCK U.S.A., INC., 790 F. Supp. 797 (E.D. Mich. 1989)................ 718

Wiwa v. Royal Dutch Petroleum Co., 226 F.3d 88 (2d Cir. 2000)......................................... 549

Zschernig v. Miller, 389 U.S. 429 (1968) .................................................................................. 10

\section{International and Foreign Cases}

Alcoa Minerals of Jamaica v. Jamaica, ICSID Case No. ARB/74/2, Dec. of July 6, 1975 ..... 649

Allianz SpA v. West Tankers Inc., ECJ Case C-185/07 OJ C 82, 48 I.L.M. 488 (2009)......... 666

Barcelona Traction, Light \& Power Co., Ltd., 1970 I.C.J. Rep. 3 .......................................... 68

Boehringer Ingelheim, KG v. Dowelhurst Ltd., Case C-348/04, OJ C 273 (Eur. Ct.

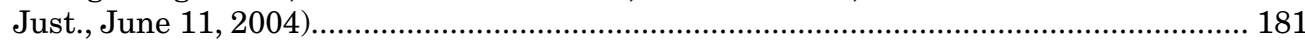

CMS v. Argentina, Decision on Juris. of July 17, 2003, 42 I.L.M. 2003 .................................. 69

DEUTSCHE BANK AG v. SRI LANKA, ICSID Case No. ARB/09/02, Award of Oct. 31, 2012 .................................................................................................................................... 70

DRAMs AND DRAM MODULES FROM KOREA, U.S. Int'l Trade Comm'n, Inv. No. 701-TA-431 (Final), Aug. 2003.......................................................................................... 454

Erich Gasser GmbH v. MISAT Srl, [2003] E.C.R. I-14693 (2003)....................................... 666

European Community - Meat and Meat Products (Hormones), WTO Doc. WT/

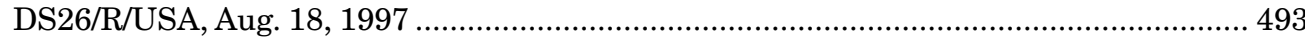

Factory at Chorzów, (Claim for Indemnity — Jurisdiction), PCIJ Judgment No. 9,

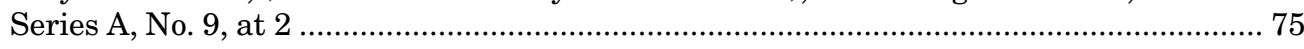

Factory at Chorzów, (Claim for Indemnity - Merits), PCIJ Judgment No. 13, Series

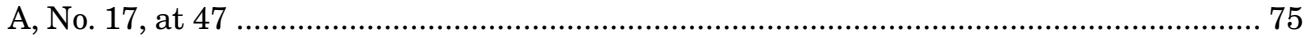

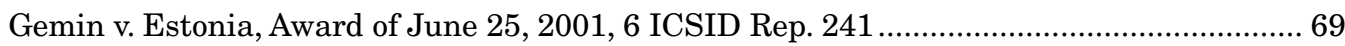

Gencor v. Commission of the European Community, Case T-102/96, 1999 E.C.R. II-753..... 532

Gesellschaft für Antriebstechnik v. Lamellen \& Kupplungsbau Beteiligungs, ECJ

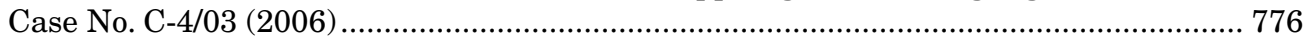

Glamis Gold, Ltd. v. United States, NAFTA Ch. 11 Arb. Trib., Award of June 8, 2009,

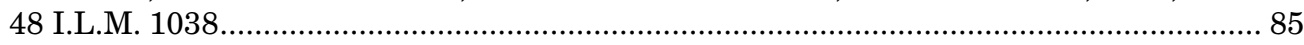

GOOGLE, INC. v. EQUUSTEK SOLUTIONS, INC., 2017 [1] S.C.R. 824 (Can.) .......... 798

Interhandel Case (Switz. v. U.S.), Prelim. Objections, 1959 I.C.J. Rep. 6 .............................. 736

Ionides v. Pacific Fire \& Marine Ins. Co., (1871) L.R., 6 Q.B. 674; (1872) L.R., 7 Q.B.

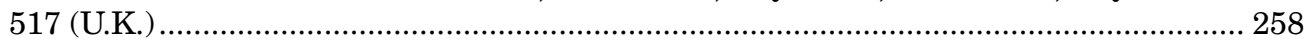

JAPAN - TAXES ON ALCOHOLIC BEVERAGES, WTO Appellate Body, Doc.

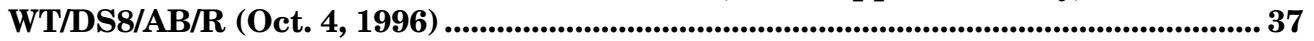

J.H. Rayner \& Co. v. Hambro's Bank Ltd., [1943] K.B. 37 (U.K.)......................................... 281 
Kadi v. Council of the European Union, Eur. Ct. Just. Case Nos. C402/05P \& C415/05P, Sept. 3, 2008

Leno Merken B.V. v. Hagelkruis Beheer B.V., Decision No. 2004448 (Benelux Off.

Intell. Prop. 2010)

Leno Merken BV v. Hagelkruis Beheer BV, Ct. Just. EU Case C149/11, Judgment of Dec. 19, 2012

LG\&E Energy Corp. v. Argentine Rep., 21 ICSID Rev. 269 (2006)

LOBSTERS FROM CANADA, U.S.-Can. Free Trade Agt. Panel, USA 89-180701, Final Rep. (May 25, 1990)

Malaysian Historical Salvors v. Malaysia, ICSID Case No. ARB/05/10, 48 ILM 1086 (2009) 62

MARVIN FELDMAN v. MEXICO, 7 ICSID Rep. 341 (2002) 79

Merck \& Co. v. Stephar BV, Case 187/80, [1992] F.S.R. 57 (Eur. Ct. Just.) ............................ 180

METALCLAD CORP. v. MEXICO, 16 ICSID Rev. 168 (2001). 85

MINE v. Guinea, Trib. 1ère Instance, Geneva, Judg. of Mar. 13, 1986, 1 ICSID Rev. 383 (Switz.) 734

MTD Equity Sdn. Bhd. v. Chile, ICSID Case No. ARB/01/7 (May 25, 2004) .......................... 90

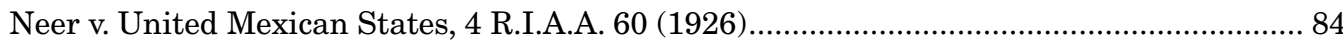

Philip Morris Brands Sarl v. Uruguay, ICSID Case No. ARB/10/7, Award of July 8, 2016 .... 85

R. v. Bow Street Metropolitan Stipendiary Magistrate ex parte Pinochet Ugarte, [2002] 1 A.C. 61 (UK)

Saipem SpA v. Bangladesh, Award of June 30, 2009, ICSID Case No. ARB/05/7, 48 I.L.M. 999 (2009)

Salini Costruttori S.p.A. v. Morocco, ICSID Case No. ARB/00/4, Decision of July 23, 2001 (Juris.)

$.62,737$

Saluka Investments v. Czech Rep., UNCITRAL Award of Mar. 17, 2006 .............................. 90

Sporrong \& Lönnroth v. Sweden, 5 E.H.R.R. 35 (1983)

Starrett Housing Corp. v. Iran, Award No. ITL-32-24-1, 4 Iran-U.S. Cl. Trib. Rep. 122 (1983)

Tokios Tokelès v. Ukraine, ICSID Case No. ARB/02/18, Dec. of Apr. 29, 2004, 20 ICSID Rev. 205 (2005)

Tokios Tokelés v. Ukraine, ICSID Case No. ARB/02/18, Order of July 1, 2003, 11 ICSID Rep. 310 (2007)

Tippetts v. TAMS-AFFA Consulting Eng. of Iran, Award 141-7-2, 6 Iran-U.S. Cl. Trib. Rep. 219 (1984).

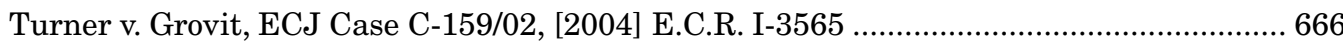

United City Merchants (Invs.) Ltd. v. Royal Bank of Can., [1983] 1 A.C. 168 (U.K.) ........... 275

United States - Cross-Border Gambling \& Betting Services, WTO Case DS285, Jan. 28, 2013

United States - Definitive Safeguard Measures on Wheat Gluten, WTO Doc. WT/ DS166/R (July 31, 2000)

United States — Import Prohibitions of Certain Shrimp, WTO Doc. WT/DS58/AB/R (Oct. 12, 1998). 
United States - Sections 301-310 of the Trade Act of 1974, WTO Doc. WT/DS152/R, Dec. 22, 1999 491

Venoklim Holdings B.V. v. Venezuela, ICSID Case No. ARB/12/22 (Apr. 3, 2015) 69

Zino Davidoff v. A\&G Imports, C-414/99, [2002] R.P.C. 20 (Eur. Ct. Just.) 181

\section{U.S. Administrative Decisions}

Bayer Consumer Care AG v. Belmora LLC, 90 U.S.P.Q.2d 1587 (T.T.A.B. 2009) 99

Certain Motor Vehicles, Inv. No. TA-201-44 (Dec. 1980), USITC Pub. 1110. 454

Fiat Grp. Auto. S.p.A. v. ISM, Inc., 94 U.S.P.Q.2d 1111 (T.T.A.B. 2010) 114 
Aaron X. Fellmeth - 9781839107429 